\title{
Microstructure of damage in thermally activated fracture of Lennard-Jones systems
}

\author{
A. Yamamoto, ${ }^{1}$ F. Kun, ${ }^{2}$ and S. Yukawa ${ }^{1}$ \\ ${ }^{1}$ Department of Earth and Space Science, Osaka University, Toyonaka 560-0043, Japan \\ ${ }^{2}$ Department of Theoretical Physics, University of Debrecen, Post Office Box 5, H-4010 Debrecen, Hungary
}

(Received 25 January 2011; published 16 June 2011)

\begin{abstract}
We investigate the effect of thermal fluctuations on the critical stress and the microstructure of damage preceding macroscopic fracture of Lennard-Jones solids under a constant external load. Based on molecular dynamics simulations of notched specimens at finite temperatures, we show that the crystalline structure gets distorted ahead of the crack in the secondary creep regime. The damage profile characterizing the spatial distribution of lattice distortions is well described by an exponential form. The characteristic length of the exponential form provides the scale of damage, which is found to be an increasing function of the temperature: At low temperatures, damage is strongly localized to the crack tip, while at high temperatures, damage extends to a broader range, leading to more efficient relaxation of overloads. As a consequence, the stress intensity factor decreases with increasing temperature. The final macroscopic failure of the system occurs suddenly and is initiated by the creation of vacancies and voids. The creep strength exhibits inverse square root scaling with the notch size corrected by the extension of the process zone.
\end{abstract}

DOI: 10.1103/PhysRevE.83.066108

PACS number(s): $62.20 . \mathrm{M}-, 46.50 .+\mathrm{a}, 81.40 . \mathrm{Np}$

\section{INTRODUCTION}

Understanding subcritical fracture occurring under constant or periodic external loads below the fracture strength of materials is a very important scientific problem with a broad spectrum of technological applications. Depending on the materials' details, several microscopic mechanisms may contribute to the time-dependent response and final failure of samples. In spite of this diversity, experiments and theoretical investigations have revealed that subcritical fracture obeys scaling relations on both macro- and microscales [1,2].

Thermal activation of microcrack nucleation and growth is one of the primary mechanisms underlying subcritical fracture. Theoretical and experimental investigations have shown that in both homogeneous and heterogeneous materials thermally driven stress fluctuations can be responsible for the finite lifetime of loaded specimens $[3,4]$, leading to the emergence of universal scaling laws such as the Andrade law and time-to-failure power laws [5,6] as well as to the scaling behavior of waiting times between microfractures [7-11]. Experiments have also revealed that thermally activated slow crack advancements affect even the surface roughness of growing cracks [12].

In order to understand the mechanisms of the subcritical fracture of crystalline and glassy materials at the atomistic scale, molecular dynamics simulations [13] performed at finite temperatures proved to be indispensable [14-19]. Computer simulations of the Lennard-Jones particle systems made possible to understand the origin of the Griffith-type crack nucleation at the atomic scale, showing the importance of the creation of vacancy clusters and voids [15-22]. The simulation technique was also extended to study dynamic fracture phenomena [23].

In the present paper, we study thermally activated subcritical fracture using molecular dynamics simulations of a Lennard-Jones particle system in two dimensions. A rectangular sample with a triangular lattice structure is constructed, which is then subject to a constant external load at different notch lengths and temperatures. We show that the critical stress of the system has an inverse square root dependence on the notch length as expected from linear fracture mechanics (LFM) corrected by the extension of the fracture process zone. The stress intensity factor at a given notch length decreases with increasing temperature due to the more efficient relaxation of overloads in the vicinity of the crack. In order to characterize the microstructure of the loaded sample, we introduce the six-fold order parameter and analyze its spatial distribution during the secondary creep regime. Computer simulations revealed that damage concentrates ahead of the crack tip and decays to a nearly homogeneous background at larger distances. The damage profile has an exponential form from which the characteristic scale of damage can be determined. When the temperature increases, the damage profile broadens and the background damage increases, which are consistent with the decreasing stress intensity factor. The results are discussed in comparison to the microstructure of heterogeneous solids with only quenched disorder under quasistatic loading just before ultimate failure [24].

\section{MODEL}

We perform molecular dynamics (MD) simulations of twodimensional solids which consists of particles. The particles interact through the Lennard-Jones (LJ) potential

$$
U(r)=4 \epsilon\left[\left(\frac{a}{r}\right)^{12}-\left(\frac{a}{r}\right)^{6}\right],
$$

where $r, \epsilon$, and $a$ are the distance between particles, the strength of the potential, and the characteristic length of the particle, respectively. For the computational efficiency, we cut the interaction potential, Eq. (1), at the distance $3 a$ when calculating the interparticle force; that is, if the distance $r$ between two particles exceeds $3 a$, the interaction disappears. The parameters $\epsilon, a$, and the mass of particles $m$ are set to be unity in all the simulations. The temperature $T$ is defined as the average kinetic energy of the system $T=\sum_{i=1}^{N} m \mathbf{v}_{i}^{2} / 2 N k_{\mathrm{B}}$, where $\mathbf{v}_{i}$ denotes the velocity of $i$ th particle, $N$ is the number 


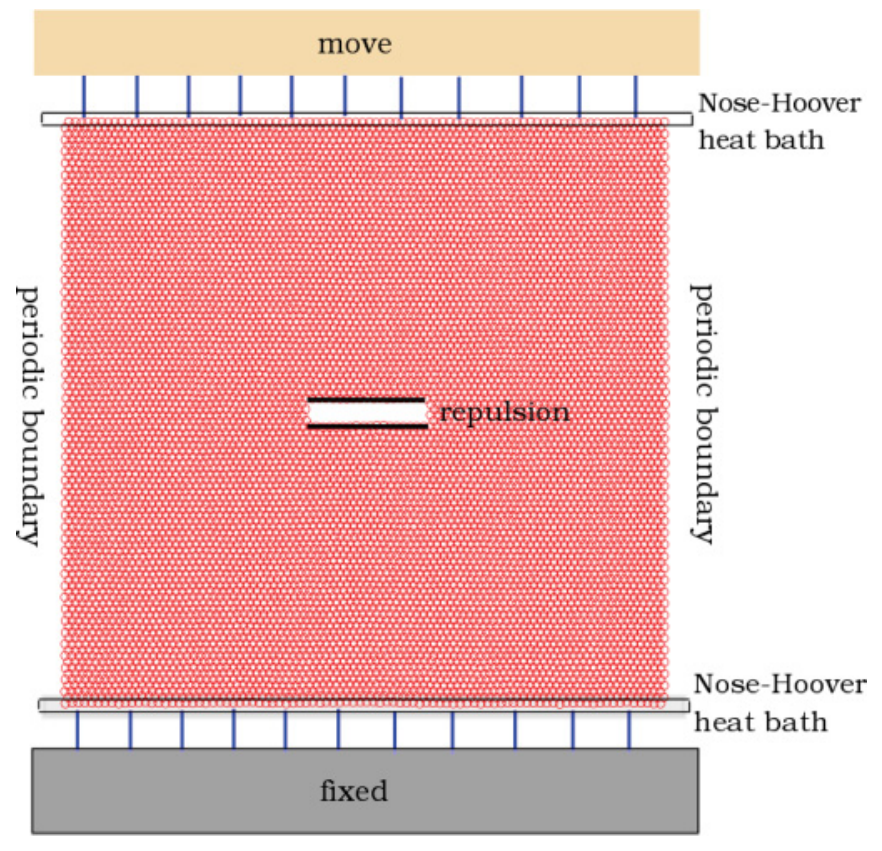

FIG. 1. (Color online) Schematic picture of the system: The sample is loaded through springs in the vertical direction. Bottom and top sides are attached to the Nose-Hoover thermostat to control the temperature. In the horizontal direction, the periodic boundary condition is applied.

of particles, and $k_{\mathrm{B}}$ is the Boltzmann's constant. Hereafter we also set the Boltzmann's constant $k_{\mathrm{B}}$ to be unity.

A schematic picture of the computational geometry is presented in Fig. 1. In order to control the temperature, Nose-Hoover thermostats are attached to two particle layers on the top and bottom of the system [25,26]. In the horizontal direction, we apply a periodic boundary condition to make the crack advance from the initial crack in the middle of the system and to prevent the vaporization of particles due to the thermal effect.

The initial state of the sample is prepared as follows: The particles are placed on a regular triangular lattice at zero temperature, $T=0$, and then the temperature of the system is slowly increased to the desired value. We take the width of the system as the corresponding equilibrium value for the temperature. Simulations were carried out with a fixed value of the width $l_{0} \simeq 100$ of the lattice. As a crucial component of the model, an initial crack is created by removing four particle layers with length $2 L$ in the middle of the system. In this way, the crack has a nearly rectangular shape with extensions $2 L \times 3.5 a$. Note that the distance $3.5 a$ of the two sides is larger than the cutoff length $3 a$ of the potential, which ensures that the removed particles form a crack in the model. In order to prevent the crack from healing due to thermal fluctuation, the particles on the upper and bottom crack faces interact through the repulsive part of the LJ potential [Eq. (1)]. After equilibrating the system, the load is applied in such a way that the bottom and top particle layers are attached to loading bars through spring elements. The bottom bar is fixed while the upper one can move vertically, controlling the load $\sigma$ on the system. The bars are treated as rigid bodies with a mass $10^{-3}$ per unit length, while the loading springs have zero mass and a spring constant of 10 . The stiffness of the solid is about 83 for the parameters used in the simulations, which is significantly larger than the one of the springs.

In order to suppress the disturbing effect of elastic waves generated by the external loading process, the loading bar moves from the initial position until the desired stress value $\sigma$ is reached at a relatively low increasing rate $d \sigma / d t=10^{-2}$. Simulations showed that a value of the loading rate slower than $d \sigma / d t=10^{-2}$ did not affect the time evolution of the system.

\section{TIME EVOLUTION}

The time evolution of the solid subject to a constant load is calculated numerically by the second-order symplectic integrator method with the time step $d t=10^{-3}$, while the time evolution of the thermostat and the bar is obtained using the Euler method. Figure 2 presents snapshots of the time evolution of the system at temperature $T=0.1$, load $\sigma=1.45$, initial crack length $2 L=20$, and width of the system $l_{0} \simeq 100$. Starting from the initial state [Fig. 2(a)], we gradually increase the external load $\sigma$ up to the desired value and equilibrate the system to achieve the desired temperature. Figure 2(b) shows the sample after the ramp loading, that is, when the stress becomes constant for the rest of the simulation. It is observed in Fig. 2(c) that the crystalline structure of the lattice gets distorted. Nucleation of defects and voids due to thermal fluctuations is also observed, which facilitates the advancement of the crack. When the specimen is separated into two parts by a crack like in Fig. 2(d), the system is considered to be fractured.

On the macroscopic level, the time evolution of the system can be monitored by measuring the vertical size $l(t)$ of the

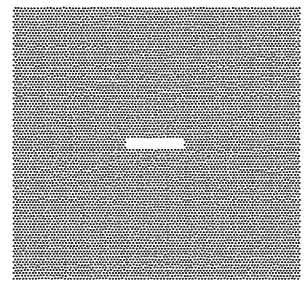

(a)

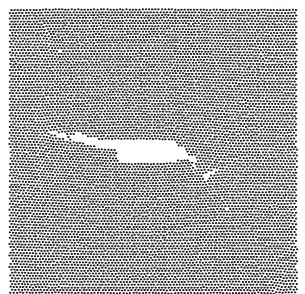

(c)

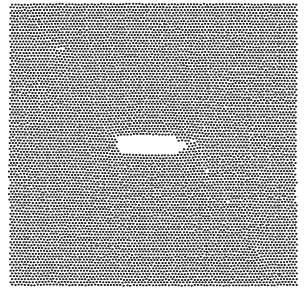

(b)

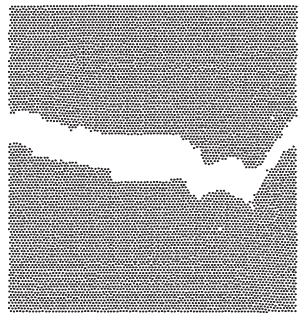

(d)
FIG. 2. Time evolutions of a sample at the temperature $T=0.1$ and the load $\sigma=1.45$ with the initial crack length $2 L=20$ : (a) initial state and (b) configuration after uploading and equilibration. (c) As time evolves, the lattice structure gets distorted, the crack grows, and voids and defects nucleate ahead of the crack tip. (d) The material's fracture occurs suddenly following a long evolution process, during which the overall deformation hardly changes. 


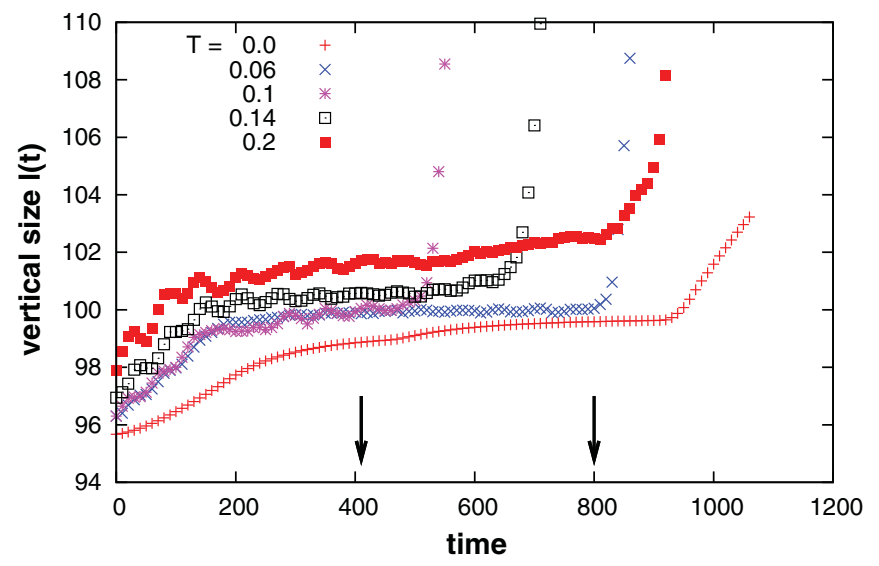

FIG. 3. (Color online) Vertical size $l(t)$ of the system as a function of time for several temperatures with the initial crack length $2 L=20$. Elastic waves generated by the loading process and the void creations due to the thermal noise cause the small oscillations of $l(t)$. The vertical arrows indicate the time window for the sample of $T=0.2$ where the damage parameter $d$ is evaluated (see Sec. V). Applied loads are taken to be $\sigma=2.05,1.7,1.45,1.35$, and 1.1 for $T=0$, $0.06,0.1,0.14$, and 0.2 , respectively.

sample in the direction of the external load as a function of time $t$. This quantity is presented in Fig. 3 for several temperatures with the initial crack length $2 L=20$. Note that the sample of Fig. 2 corresponds to the curve of $T=0.1$ in Fig. 3. The plateau regime of the deformation is the consequence of the slow dynamics of the system, where the lattice structure gets gradually distorted while the length of the crack hardly changes. The final macroscopic failure occurs rapidly, which is characterized by the sudden acceleration of the vertical size $l(t)$ and by its diverging derivative in Fig. 3. The acceleration of the vertical size is preceded by the creation of defects and voids due to thermal activation. Small oscillations are observed along the plateau of $l(t)$, which are caused by elastic waves generated by the loading process and the void creations due to the thermal noise.

\section{MACROSCOPIC STRENGTH}

The main difficulty of the investigation of thermally activated fracture of LJ systems is the enormous computational time required due to the slow dynamics of the system. To overcome this problem, we introduce a threshold time of the simulation; After the stress $\sigma$ reaches the desired value, we limit the simulation time to the finite threshold time $t_{\mathrm{th}}$. This threshold gives a lower limit of the lifetime of the sample: If no complete failure is achieved by following the time evolution of the system up to $t_{\text {th }}$, the lifetime of the sample is assumed to be infinite at the given external load $\sigma$. The highest load at which no macroscopic failure occurs before $t_{\text {th }}$ defines the critical load $\sigma_{c}$ of the system which separates the regimes of finite and infinite lifetimes. The critical load $\sigma_{c}$ of the system is determined by a sequence of simulations performed with a constant load increment 0.05 , which provides sufficient accuracy for $\sigma_{c}$. In this paper, the threshold time is fixed to be $t_{\text {th }}=10^{3}$.
In order to investigate the effects of the notch size $2 L$ and the temperature on the macroscopic strength $\sigma_{c}$, we carry out simulations with the following parameters: $2 L=0,5,10,15$, $20,25,30,35,40$, and 45 for the temperatures $T=0,0.06$, $0.1,0.14$, and 0.2 . We do not show the data of $2 L=0$ and 5 at $T=0$ because the crack did not grow at the crack tip. The value of $\sigma_{c}$ is determined by averaging over 10 samples at each parameter set.

Based on linear fracture mechanics, we expect the inverse square root dependence of the critical load $\sigma_{c}$ on the notch size $L$ as it has been proved by an analytical calculation [27]. In order to capture the disordering effect of temperature, we introduce a characteristic length $\xi$ which modifies the notch size into an effective one, $L+\xi$. Then we write $\sigma_{c}$ in the following form:

$$
\sigma_{c}(L, T)=\frac{K}{\sqrt{L+\xi}}+c,
$$

where $K$ denotes the stress intensity factor [24,27] and $c$ is a finite size correction of the system width. The finite size correction $c$ is a negative value, and its system size dependence is plotted in the inset of Fig. 4. It is important to emphasize that the value of the correction factor $c$ goes to zero with increasing system size $l_{0}$. To numerically demonstrate the validity of the functional form Eq. (2), in Fig. 4 we plot $\left[1 / \sigma_{c}^{\prime}(L)\right]^{2}$, where $\sigma_{c}^{\prime}(L)=\sigma_{c}(L)-c$, as a function of half of the notch length $L$. It is observed in Fig. 4 that straight lines are obtained with a good accuracy at all temperatures by fitting. Deviations from the linear form are observed for both large and small values of the initial crack length: In the large crack length regime $20 \lesssim L$, deviations occur due to the finite size of the system; on the other hand, in the limit of small cracks $L \lesssim 2.5$, the

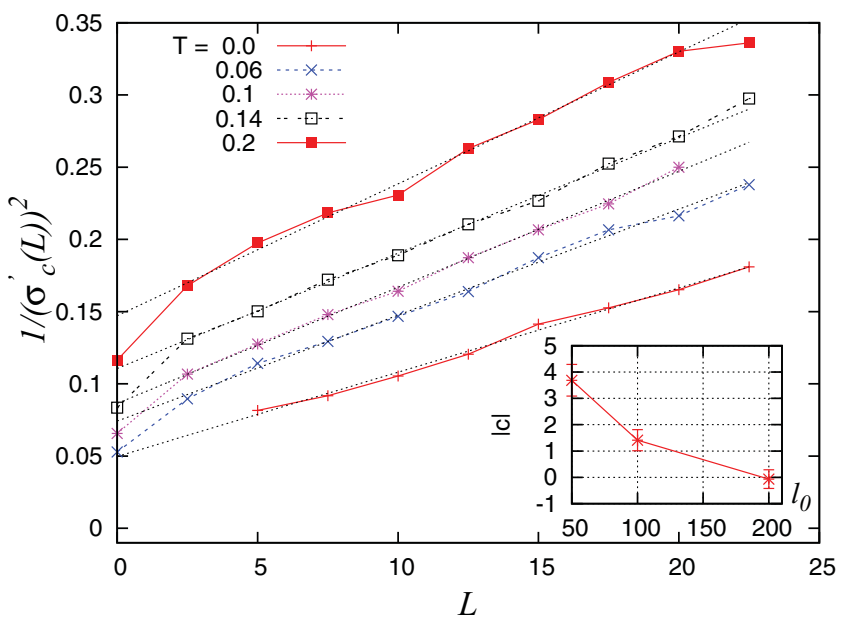

FIG. 4. (Color online) Crack length dependence of the critical stress: $\left[1 / \sigma_{c}^{\prime}(L)\right]^{2}$, where $\sigma_{c}^{\prime}(L)=\sigma_{c}(L)-c$ and $c$ is a finite size correction, is plotted as a function of $L$ for several temperatures averaging over 10 samples. The error bars are smaller than the symbol size. The dotted lines are obtained by fitting straight lines in a good agreement with Eq. (2). Deviations near $L>20$ of the data from the linear fit occur due to finite size effects, while the deviations for the small notch size indicate the dominance of thermal noise in the fracture process. Inset: System width $l_{0}$ dependence of the finite size correction $c$. 


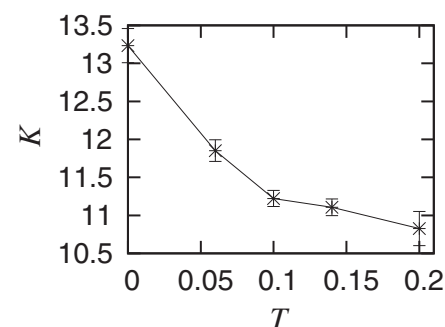

(a)

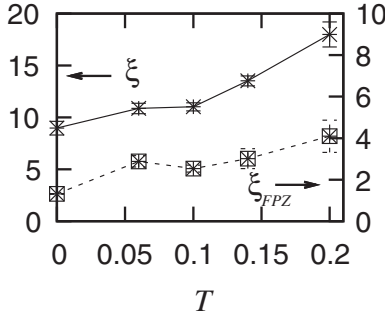

(b)
FIG. 5. (a) Temperature dependence of the stress intensity factor $K$ : As the temperature increases, the value of $K$ monotonically decreases. (b) Solid line shows the temperature dependence of the characteristic length $\xi$ defined by Eq. (2). Dashed line represents the extension of the distorted zone $\xi_{\mathrm{FPZ}}$ determined by the analysis of the microstructure of the system. (This analysis is carried out in Sec. V.) Simulations are carried out with a fixed notch size $2 L=20$.

stress concentration at the crack tip becomes less dominating so that the fracture process is mainly controlled by thermal noise emerging in the entire volume of the system. By fitting these data from $L=2.5$ to 20 , we estimate the stress intensity factor $K$ and the characteristic length $\xi$ of Eq. (2) for each temperature.

Figures 5(a) and 5(b) present the temperature dependence of the stress intensity factor $K$ and $\xi$, respectively. It is observed that the stress intensity factor $K$ decreases with increasing temperature $T$, while the value of $\xi$ is an increasing function of $T$. In Fig. 5(a), at higher temperature thermally activated particle motion has a higher intensity which facilitates the relaxation of the system and gives rise to a decreasing $K$. In Fig. 5(b), the results imply that at higher temperature a broader zone is formed ahead of the crack tip where distortion of the crystalline structure and plastic deformation can occur due to the interplay between the stress field and the thermal noise. The length scale $\xi$ can be interpreted as the linear extension of the fracture process zone (FPZ) ahead of the crack tip, which appears due to the disordering effect of thermal noise on the lattice structure. In the vicinity of the crack tip, the high stress concentration makes the system more sensitive to thermal fluctuations. As a consequence, a distorted zone emerges which affects the scaling of $\sigma_{c}$ with the notch size $L$. A similar effect is observed in heterogeneous quasibrittle materials where damage in the form of microcracks is concentrated ahead of the crack [24,27].

\section{MICROSTRUCTURE OF DAMAGE}

In order to obtain a direct quantitative measure of the extension of the process zone formed ahead of the crack tip, we carry out a detailed analysis of the microstructure of the system before the acceleration of deformation starts. To characterize the crystalline order, we introduce the bond-orientational order parameter $\phi_{k}$ defined for particle $k$ as follows [28]:

$$
\phi_{k}=\left|\sum_{l}^{n_{k}} \frac{\exp \left(6 i \theta_{k l}\right)}{n_{k}}\right| \text {. }
$$

Here $n_{k}$ denotes the number of neighbors of particle $k$ and $\theta_{k l}$ is an angle between a fixed axis such as the $x$ axis and the bond connecting particle $k$ to particle $l$. The value of $\phi_{k}$ can vary between zero and one such that $\phi_{k}=1$ quantifies a perfectly ordered triangular lattice, while $\phi_{k} \approx 0$ is obtained for a disordered fluidlike system. Intermediate values of the order parameter characterize the degree of distortion of the lattice structure.

In order to characterize the spatial variation of the microstructure, we calculate a locally averaged bondorientational order parameter $\phi$ on a coarse-grained mesh in the sample. The mesh size is taken to be $2 \times 2$. The order parameter is evaluated for a single sample, but 30 snapshots are taken from the plateau regime of the deformation-time diagrams in order to improve the statistics. As shown in Fig. 3, the two vertical arrows indicate the time window of the plateau regime for the case of temperature $T=0.2$. A typical example of the order parameter $\phi$ for the temperature $T=0.06$ is presented in Fig. 6. It is observed that the crystalline structure has strong distortion in the vicinity of the crack tip where the stress concentration is high. Away from the crack tip, and even near the middle of the crack, the order parameter is homogeneously distributed and it has a large value close to unity; that is, the crystalline structure is retained there.

To obtain a detailed quantitative measure of the disordering effect of the stress concentration assisted by the thermal noise, we introduce a damage parameter with the following definition:

$$
d_{k}=1-\phi_{k},
$$

which has a large value $d_{k} \approx 1$ at locations where the lattice structure is most distorted. Based on the above meshing technique, we average $d_{k}$ on each plaquette of the mesh, then make a projection on the horizontal axis and determine the damage parameter along the initial crack axis as a function of the distance from the crack tip $x$. At each position $x$,

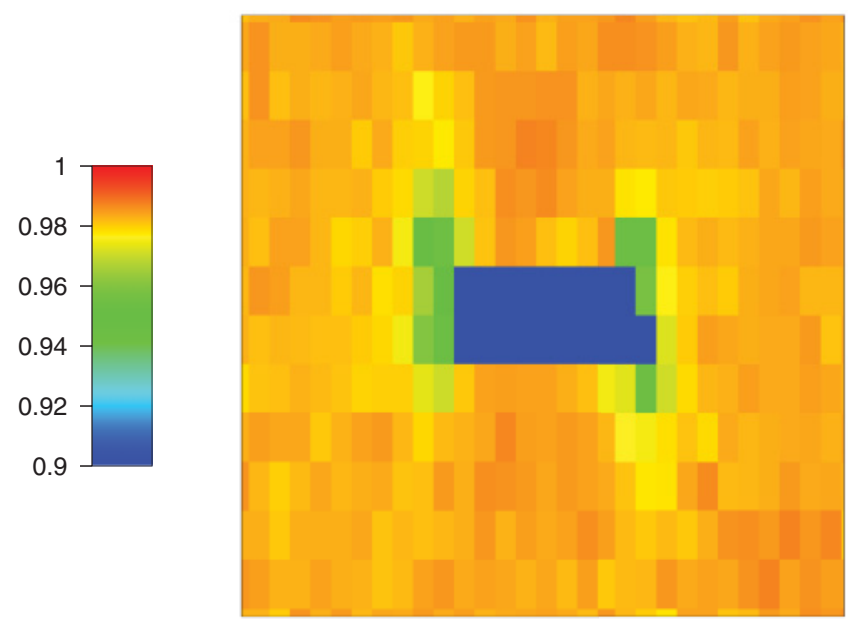

FIG. 6. (Color online) Spatial distribution of the bondorientational order parameter averaged on a rectangular $2 \times 2$ mesh: The temperature and the initial crack length are taken to be $T=0.06$ and $2 L=20$. For the sake of clarity, we present a magnified view of the vicinity of the crack. The color code indicates the modification of the triangular-crystalline structure due to the high stress concentration and to the thermal noise. Deep blue (black in print) indicates the order parameter less than 0.9. In this figure, the blue (black) region corresponds to the crack. 


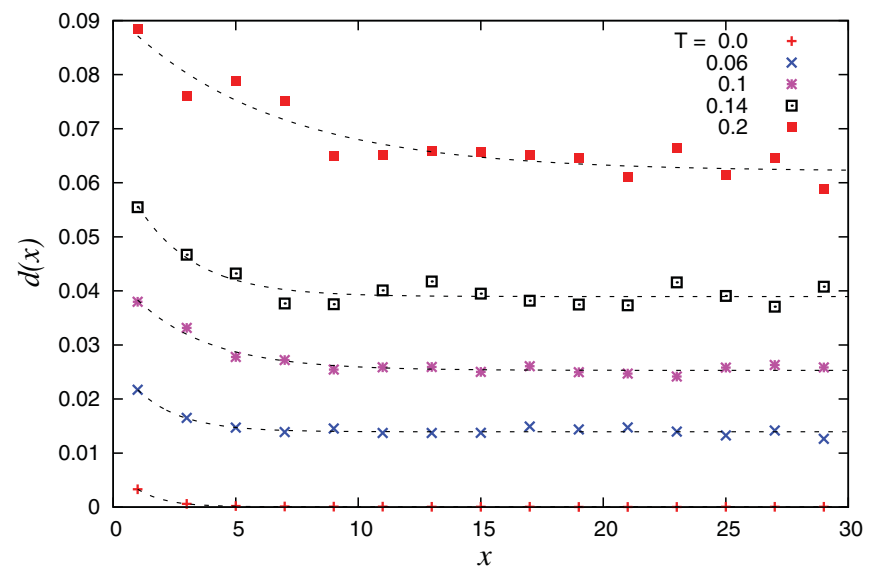

FIG. 7. (Color online) Damage profile, that is, the damage parameter $d$ as the function of the distance $x$ from the crack tip for the initial crack length $2 L=20$ at several temperatures. The damage profile exhibits an exponential decay with the form Eq. (5) to a uniform background damage. All the three parameters characterizing the functional form of $d(x)$ depend on the temperature [see Figs. 5(b) and 8 ].

the averaged damage parameter is averaged again on the vertical column of five plaquettes. The damage profile $d(x)$ obtained by this way is presented in Fig. 7 for several different temperatures. Note that the calculations are performed at the same load value $\sigma=1.0$ for all the temperatures at the given notch length $2 L=20$. It is observed that $d(x)$ monotonically decreases with increasing distance $x$ from the crack tip as expected and converges to finite values at long distances. The figure shows that the profiles can be well described by the exponential form

$$
d(x)=A+B \exp \left(-x / \xi_{\mathrm{FPZ}}\right),
$$

where the parameter $A$ represents the uniform background damage, $\xi_{\text {FPZ }}$ is the characteristic length of the extension of the distorted zone, and the multiplication factor $B$ represents the maximum value of the additional damage above the background achieved at the crack tip. The functional form, Eq. (5), provides an excellent fit of the numerical data in all cases. Therefore, we determine the temperature dependence of the parameters $A, B$, and $\xi_{\text {FPZ }}$ [see Figs. 8 and 5(b)]. Note that the damage parameter $d$ at zero temperature decreases to zero at large distances, while it takes a nonzero value at the crack tip. This clearly indicates that the distortion of the lattice is caused by the high stress concentration arising in the vicinity of the crack tip. By increasing the temperature, the thermal noise gets stronger, which lightens the stress concentration near the crack tip by distorting the lattice structure. This mechanism has the consequence that with increasing temperature the localization of stress at the crack tip decreases, which is quantified by the increasing characteristic length $\xi_{\text {FPZ }}$ [see Figs. 5(b) and 8].

The most important characteristic of the microstructure is represented by the length scale $\xi_{\mathrm{FPZ}}$, which provides a measure of the extension of the highly distorted zone. It can be observed in Fig. 5(b) that $\xi_{\mathrm{FPZ}}$ increases when the temperature gets higher. It shows that increasing thermal noise in the system gives rise to a larger extension of the fracture process zone. It can also be observed in Fig. 5(b) that $\xi$ and $\xi_{\text {FPZ }}$ have a linear

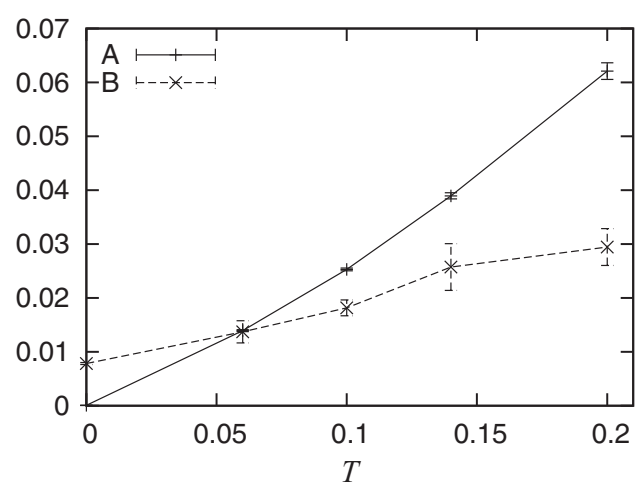

FIG. 8. The parameters $A$ and $B$ of the damage profile as function of temperature for the notch size $2 L=20$. The value of the background disorder $A$ rapidly increases with the temperature, while the multiplication factor $B$ has a moderate increase.

relation, which implies that the microstructure-based length $\xi_{\text {FPZ }}$ provides a good measure of the macroscopic fracture process zone.

\section{CONCLUSIONS AND DISCUSSION}

We carried out a detailed study of the creep rupture of Lennard-Jones particle systems by means of molecular dynamics simulations. In order to control the temperature, a Nose-Hoover thermostat was coupled to the sample, which was then subject to a constant load through spring elements. The main focus of our work was to understand the effect of thermal fluctuations on the creep strength and to investigate the microstructure of the evolving system before the onset of rapid crack growth, leading eventually to macroscopic failure. Simulations showed that the critical stress of samples has an inverse square root dependence on the notch length corrected by the extension of the fracture process zone. The fracture process zone arises due to the distortion of the lattice structure as a consequence of the interplay of the high stress concentration at the crack tip and the thermal noise. The final failure occurs rapidly after the creation of vacancies and voids in the process zone.

In order to obtain a direct quantitative measure of the extension of the fracture process zone, we introduced a damage parameter and analyzed its spatial distribution. Our calculations revealed that damage concentrates in the vicinity of the crack tip and decays exponentially to a homogeneous background damage level. In spite of the inverse square root form of the stress distribution ahead of the crack expected from linear fracture mechanics, the damage profile is described by an exponential form, which let us introduce a characteristic length scale of damage $\xi_{\mathrm{FPZ}}$. The highly distorted regime of extension $\xi_{\mathrm{FPZ}}$ is analogous to the fracture process zone observed in various types of systems.

A similar exponential form of the damage profile was recently found for heterogeneous materials where thermal activation does not play a role [24]. The authors investigated quasibrittle fracture processes in the framework of the fuse model, gradually increasing the load on notched samples up to failure. When evaluating the density of microcracks in the critical state of the sample just before macroscopic failure, it 
was found that damage concentrates ahead of the crack. The damage profile proved to have an exponential form similar to ours. It was argued that microcracks concentrate at the crack tip due to the high stress concentration; however, as a countereffect, they screen the stress field, which then leads to the exponential decay instead of the inverse square root form [24].

A very interesting question addressed by Ref. [24] is the crossover between the two regimes where the fracture strength of the system is dominated by stress concentration at the notch and by the disorder, respectively. The deviation from the linear behavior in Fig. 4 at the limit of small notch sizes already indicates the dominance of thermal noise in the fracture process. However, due to technical reasons, we could not further decrease the initial crack length, which prevented us from deducing a quantitative conclusion on the crossover phenomenon.

\section{ACKNOWLEDGMENTS}

We acknowledge support of the MTA-JSPS Project 32/2008, the TeT Project JP-24/09, the Complexity-Net, and Grant-in-Aid for Scientific Research (C) No. 22540387 from the Ministry Education, Culture, Sports, Science, and Technology Japan. The work is supported by the TAMOP-4.2.1/B09/1/KONV-2010-0007 Project. The project is implemented through the New Hungary Development Plan, cofinanced by the European Social Fund and the European Regional Development Fund. F. Kun acknowledges support of the Janos Bolyai Project of HAS and of OTKA K84157. A. Yamamoto acknowledges support of the Global COE Program (Core Research and Engineering of Advanced MaterialsInterdisciplinary Education Center for Materials Science), MEXT, Japan, and the JSPS International Training Program (ITP).
[1] M. J. Alava, P. K. V. V. Nukala, and S. Zapperi, Adv. Phys. 55, 349 (2006).

[2] J. Rosti, J. Koivisto, L. Laurson, and M. J. Alava, Phys. Rev. Lett. 105, 100601 (2010).

[3] D. Bonn, H. Kellay, M. Prochnow, K. Ben-Djemiaa, and J. Meunier, Science 280, 265 (1998).

[4] A. Guarino, A. Garcimartin, and S. Ciliberto, Eur. Phys. J. B 6, 13 (1998)

[5] A. Saichev and D. Sornette, Phys. Rev. E 71, 016608 (2005).

[6] H. Nechad, A. Helmstetter, R. El Guerjouma, and D. Sornette, Phys. Rev. Lett. 94, 045501 (2005).

[7] A. Guarino, L. Vanel, R. Scorretti, and S. Ciliberto, J. Stat. Mech. P06020 (2006).

[8] P.-P. Cortet, L. Vanel, and S. Ciliberto, Phys. Rev. Lett. 99, 205502 (2007)

[9] S. Ciliberto, A. Guarino, and R. Scorretti, Physica D 158, 83 (2001).

[10] S. Santucci, L. Vanel, and S. Ciliberto, Phys. Rev. Lett. 93, 095505 (2004).

[11] L. Vanel, S. Ciliberto, P.-P. Cortet, and S. Santucci, J. Phys. D 42, 214007 (2009).

[12] N. Mallick, P.-P. Cortet, S. Santucci, S. G. Roux, and L. Vanel, Phys. Rev. Lett. 98, 255502 (2007).

[13] M. P. Allen and D. J. Tildesley, Computer Simulation of Liquids (Oxford Science Publications, 1987).
[14] N. Yoshioka, F. Kun, and N. Ito, Phys. Rev. Lett. 101, 145502 (2008).

[15] C. L. Dias, J. Kröger, D. Vernon, and M. Grant, Phys. Rev. E 80, 066109 (2009).

[16] L. Golubovic and A. Peredera, Phys. Rev. E 51, 2799 (1995).

[17] L. Golubovic, A. Peredera, and M. Golubovic, Phys. Rev. E 52, 4640 (1995).

[18] M. Adda Bedia, R. Arias, M. Ben Amar, and F. Lund, Phys. Rev. E 60, 2366 (1999).

[19] C. D. Lorenz and M. J. Stevens, Phys. Rev. E 68, 021802 (2003).

[20] K. S. Cheung and S. Yip, Phys. Rev. Lett. 65, 2804 (1990).

[21] M. Ippolito, A. Mattoni, L. Colombo, and N. Pugno, Phys. Rev. B 73, 104111 (2006).

[22] A. Mattoni, L. Colombo, and F. Cleri, Phys. Rev. Lett. 95, 115501 (2005).

[23] M. Marder, Int. J. Fract. 130, 517 (2004).

[24] M. J. Alava, P. K. V. V. Nukala, and S. Zapperi, Phys. Rev. Lett. 100, 055502 (2008).

[25] W. G. Hoover, Phys. Rev. A 31, 1695 (1985).

[26] S. Nose, J. Chem. Phys. 81, 511 (1984).

[27] Z. Bazant and J. Planas, Fracture and Size Effect in Concrete and Other Quasibrittle Materials (Boca Raton and London: CRC Press, 1998) ISBN 0-8493-8284-X.

[28] B. I. Halperin and D. R. Nelson, Phys. Rev. Lett. 41, 121 (1978). 